\title{
A Novel Study on the Power Output Characteristics of CT Power Supply
}

\author{
Changtao Chen ${ }^{1}$, Jie Lou ${ }^{1}$, Yuying Zhang ${ }^{1}$, Ruochen Guo ${ }^{1}$ and Lei Duan ${ }^{2}$ \\ ${ }^{1}$ School of Electrical Engineering, Shandong University, Jinan, China \\ ${ }^{2}$ State Grid Weifang Power Supply Company, Weifang, China
}

\begin{abstract}
The power supply based on current transformer (CT) is a good method to provide energy for online monitoring devices. In order to study the power output characteristics of CT power supply, a simulation model is built under Saber software. Find that the output power of CT first increases and then decreases with the increase of the filter capacitor voltage. There is a maximum point and the maximum power is independent of turn number when voltage drop of rectifier is ignored. When considering the voltage drop, the maximum power increases with the increase of turn number, but becomes saturated in the end. In order to improve the efficiency of the CT power supply and reduce current dead zone, the operating point should be near the maximum power output point. Take the minimum output power of $0.5 \mathrm{~W}$ as an example, the turn number is selected and the corresponding circuit is designed. The correctness of the analysis is verified by experiments.
\end{abstract}

Keywords-current transformer; power output characteristic; rectifiers; saber; turn number

\section{INTRODUCTION}

With the development of smart grid, more and more online monitoring devices have been applied to grid monitoring. But because of the installation position and operating environment, to provide stable and reliable power supply is a technical problem. At present, the common methods used are solar cell[1], CT (current transformer) [2,3] and so on. But the output power of the solar cell can be influenced by the weather, and its size is large, installation is not convenient, battery life is short. The using of CT as the power supply has the characteristics of small size, convenient installation and large output power. As a result, it has a good application prospect and is widely concerned by scholars.

Because of the fluctuation of the line current, the CT power supply has the problems such as the iron core is easy to be saturated, the output power is small when the line current is small. In order to solve the problems, many scholars have done some research on it. For example, by adding air gap to suppress the magnetic saturation in the case of large line current[4]; To suppress the iron core saturation by adding the auxiliary protection winding [5]; Through the way of CT with battery, the battery is used as auxiliary power supply when the output power of CT is low[6]; By using the nanocrystalline material which has high permeability as the core, to improve the output power in the case of small current and reduce the dead zone[7]. These studies have solved some of the problems.
However, in previous studies, the output characteristics of CT when is connected to the actual voltage conversion circuit are less studied. The general form of the voltage conversion circuit consists of rectifier, filter and DC-DC circuit. Due to the characteristics of the fixed output voltage of the DC-DC circuit, the output power is fixed when its output port is connected with a fixed load. The input current of DC-DC circuit will reduce due to the increase of the input voltage, or increase due to the decrease of the input voltage. And the output voltage and current of CT are no longer sinusoidal form. This is different from the characteristics of the CT directly connected to the resistive load. The output current and voltage have positive correlation when CT is directly connected with the resistive load. So it is necessary to discuss the output characteristics of CT when it is connected to the actual voltage conversion circuit. This paper will discuss the power output characteristics of the CT when it connects the rectifier, filter and the DC-DC circuit. Then give the turn number selection method and design corresponding circuit.

\section{RELATIONSHIP BETWEEN OUTPUT POWER OF CT AND FILTER CAPACITOR VOLTAGE}

When the CT is directly connected to the resistance load, the theory proves that there is a maximum power output point. When the core loss is neglected, the maximum output power is determined by the following formula[8]:

$$
P_{\max }=\pi \mu f S I_{1}^{2} /
$$

Where $\mu$ is magnetic permeability, $S$ is core area, $l$ is average length of magnetic path, $f$ is current frequency, $I_{l}$ is line current (RMS).

It can be known from the formula (1) that the maximum power is only related to the primary side current, core material and size, but has nothing to do with the turn number. When the secondary side coil of CT is connected with the bridge rectifier and capacitor filter circuit, the output characteristics are different from that of the directly connected resistance load. In order to study the power output characteristics of this kind of situation, the model was built and the simulation was carried out under the Saber software.

The simulation circuit is shown in Figure I, use the nonlinear transformer model as the equivalent of CT, and its primary side coil is 1 turn and flows through a constant sinusoidal current. 
The core material is $3 \mathrm{C} 8$ ( $\mathrm{MnZn}$ ferrite), the magnetic path length is $0.212 \mathrm{~m}$, the magnetic path area is $8.25 \mathrm{~cm}^{2}$ and the filter capacitance is $5000 \mathrm{uF}$. When the circuit reaches to steady state, the filter capacitor voltage is stable, at this time, the output power of CT is equal to the power consumption of resistive load. The output power of the CT is obtained by calculating the power consumption of the resistor. Then the relationship between the output power of CT and the filter capacitor voltage is obtained by changing the load resistance.

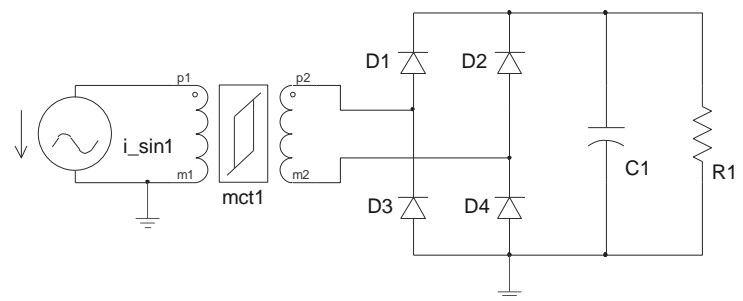

FIGURE I. OUTPUT CHARACTERISTIC SIMULATION CIRCUIT

Because the voltage drop of the rectifier can affect the results, especially when the output voltage of the CT is low. So set the voltage drop of the diode to 0 volt. The situation when the primary current is $5 \mathrm{~A}$ RMS and 10A RMS is simulated. The results are shown in Figure II. In Figure II (a) from left to right, turns are 50, 80, 120, 150, 180, 210, 250, 300. And in Figure II (b) from left to right, turns are 100,150, 200, 250, 300. As can be seen from the simulation results, the output power of CT increases first and then decreases with the increase of filter capacitor voltage. There is a maximum power point, and the voltage corresponding to the maximum power point is related to the turn number and primary side current. The maximum output power of the CT is independent of the turn number (ignore the voltage drop of the rectifier).

When the voltage drop of the rectifier is considered, the simulation results are shown in Figure III when the primary current is 5A RMS. In the figure, from left to right, the turn number is $100,150,200,250,300,350,400$. As can be seen from the figure, the change trend of the output power is the same. But the output power is low when the turn number is low. The reason is that the output voltage of CT is lower when the turn number is less, the influence of the rectifier loss is larger, so the output power is low. With the increase of the turn number, the influence of the rectifier loss becomes smaller, and the output power is increased. But the output power does not increase infinitely with the increase of turn number. In the end, it is close to the case when the voltage drop of the rectifier is ignored in Figure II.

The relationship between the maximum output power of CT and turn number is shown in Figure IV. It can be known that the maximum output power of CT will increase with the increase of turn number due to the influence of the voltage drop of the rectifier. This phenomenon is more obvious when the line current is small and the output voltage of CT is low. When the line current is small, in order to make the CT output more power, the turn number should be optimized. The maximum output power increases with the increase of turn number, but the trend is slow. It is needed to choose a reasonable turn number, so that the maximum output power is close to the ideal value, while the turn numbers are not too many.

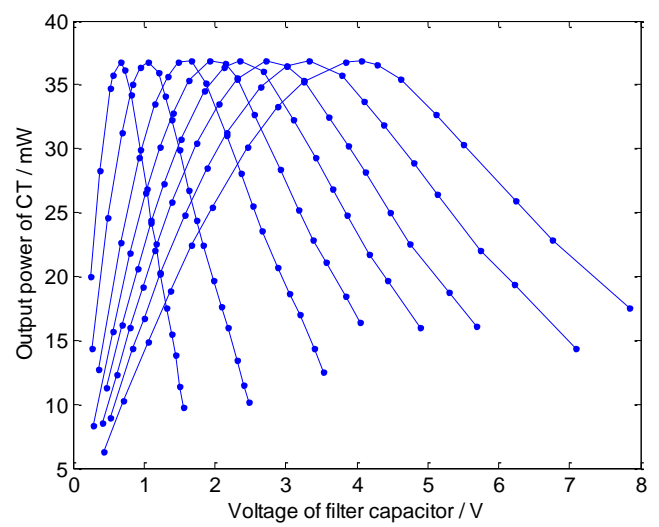

(a) $\mathrm{I} 1=5 \mathrm{~A}$ RMS

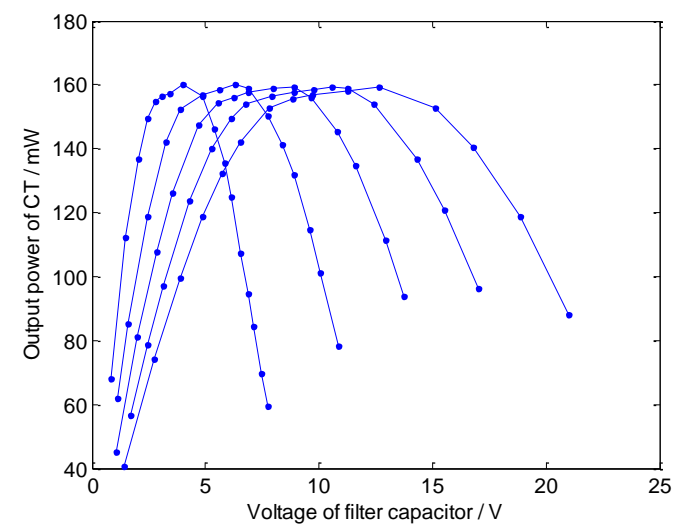

(b) $I 1=10 \mathrm{~A}$ RMS

FIGURE II. RELATIONSHIP BETWEEN OUTPUT POWER OF CT AND FILTER CAPACITOR VOLTAGE (IGNORE VOLTAGE DROP OF RECTIFIER BRIDGE)

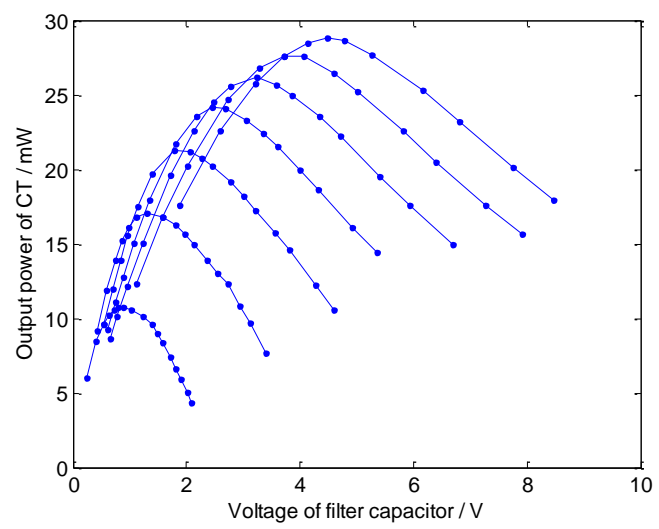

FIGURE III. RELATIONSHIP BETWEEN OUTPUT POWER OF CT AND FILTER CAPACITOR VOLTAGE (CONSIDER VOLTAGE DROP OF RECTIFIER BRIDGE) 


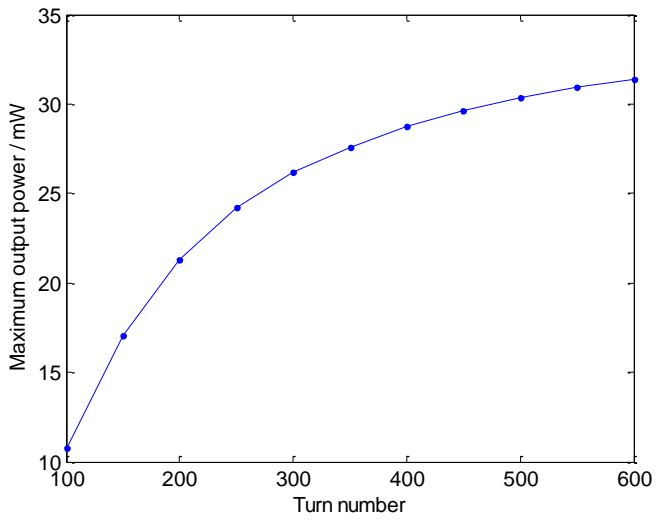

FIGURE IV. RELATIONSHIP BETWEEN MAXIMUM OUTPUT POWER AND TURN NUMBER

\section{POWER OUtPut CHARACTERISTICS OF CT POWER SUPPLY}

Considering the output power of CT increases first then decreases with the increase of filter capacitor voltage. For the same power output point, the output voltage may be different, such as point A and B in Figure V. The capacitor voltage of point A is low, and the output current of CT is high. On the contrary, the capacitor voltage of point $\mathrm{B}$ is high, and the output current of CT is low. But it can be known by analyzing that point $\mathrm{A}$ is unstable, point $\mathrm{B}$ is stable. For fixed power consumption, when the voltage of point B appears small fluctuations, the working point will return to B. For example, if the voltage drops a little, the output power of CT will increase, then the voltage will rise to point $\mathrm{B}$, or if the voltage increases a little, the output power of CT will decrease, then the voltage will decrease to point $\mathrm{B}$. However, point $\mathrm{A}$ is on the contrary, when the voltage of point A appears small fluctuations, the working point will deviate from the point A. The voltage will possibly drop to a lower value, or increase to point B. Then it can be known that the area I in Figure V is an unstable area, and the area II is a stable area.

When the CT power supply is working properly, it should be in the area II. At this time the whole circuit is in an automatic power balance state. With the load power consumption fluctuations, the balance point moves on the voltage power curve. When the load power consumption is reduced, the balance point moves to the right (voltage increasing direction), until it reaches a new balance of power. When the load power consumption increases, the balance point moves to the left (voltage drop direction). Before reaching the maximum power output point, the circuit is able to maintain the balance. When the maximum power output point is still unable to maintain the balance of power, it shows that the line current cannot maintain the power consumption of the load at this time. After crossing the maximum power point, the circuit is no longer balanced. With the decrease of the output power of CT, the filter capacitor voltage drops rapidly. When the voltage is lower than the minimum input voltage required by the DC-DC circuit, the DC-DC circuit does not work properly. It loses the voltage regulating ability, and its output voltage decreases. At this time the output power of the DC-DC circuit (or load power consumption) decreases too, and in the end, the filter capacitor voltage is stable at a lower value (below the minimum input voltage of the DC-DC circuit).

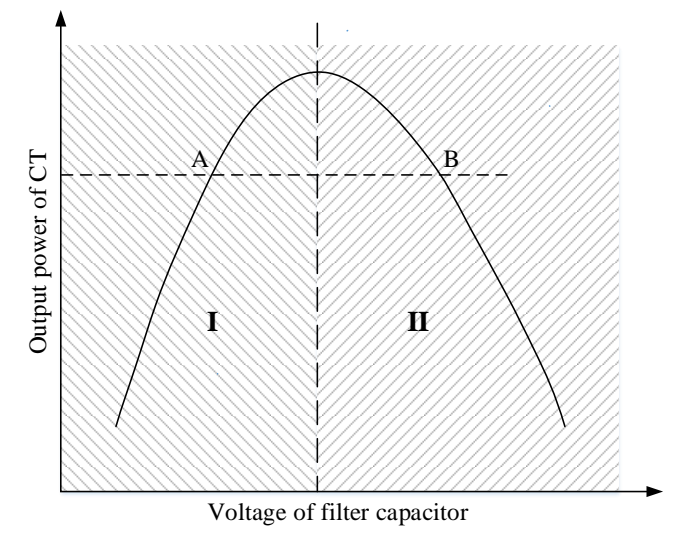

FIGURE V. OUTPUT VOLTAGE POWER CURVE OF CT

\section{CT POWER SUPPLY DESIGN}

\section{A. CT Turn Number Determination}

In order to reduce the current dead zone of CT, the CT should output power as more as possible when the line current is small. Considering the effect of magnetic permeability and saturated flux density on the output power of CT, in this paper, select the silicon steel as the core material. Take the temperature monitoring device as an example, its normal working power is generally not more than $0.5 \mathrm{~W}$. This paper chose the ring type core and its size is $40 \times 95 \times 30 \mathrm{~mm}$ (inner diameter, outer diameter, height). The design goal is that the output power is more than $0.5 \mathrm{~W}$, and at this time, the line current is as small as possible. In order to determine the reasonable turn number, the model of the CT is established under the Saber software with its MCT (Magnetic Component Tool). The magnetization curve of the core is described by Jiles-Atherton model[9].

As the maximum power output point is the critical stable point, in fact, the circuit cannot be stable for a long time at the maximum power output point. Taking into account the conversion efficiency of the actual circuit and the power consumption of the circuit itself, the simulation results should be kept in a certain margin. When the target output power is $0.5 \mathrm{~W}$, the simulation results should be larger than it. The simulation results when the line current is 10A RMS are shown in Figure VI, Its maximum power is more than $1 \mathrm{~W}$. The curves from left to right, the turn number is 60, 80, 100, 120, 160, 200.

From the figure we can know that, with the increase of turn number, the maximum output power of CT changes small. This is because the silicon steel core has high permeability, the output voltage of CT is high. Due to the limitation of the maximum input voltage of the DC-DC circuit, in this design, the maximum input voltage is $40 \mathrm{~V}$. The turn number needs to meet the condition that the filter capacitor voltage corresponding to maximum power point is less than $40 \mathrm{~V}$. In order to make the CT work properly when the line current reaches to $1000 \mathrm{~A}$, the turn number of CT cannot be too small. Otherwise, the discharge of 
secondary side coil will flow through a large current, which will lead to serious heating of the rectifier and MOSFET. According to Figure VI, select 120 turns. When the line current is less than $10 \mathrm{~A}$, the CT can reach to the maximum power output point. At the same time, when the line current reaches to $1000 \mathrm{~A}$, the output current of the secondary side is about 8.3A, which is in the range of the circuit.

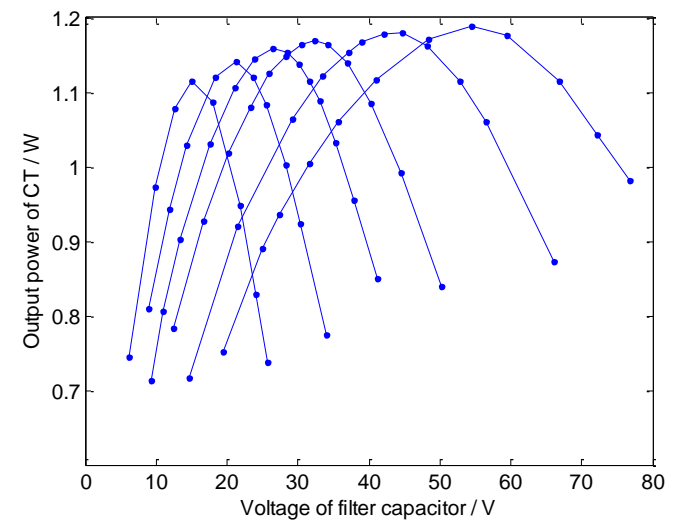

FIGURE VI. RELATIONSHIP BETWEEN OUTPUT POWER OF CT AND FILTER CAPACITOR VOLTAGE

\section{B. Design of Hardware Circuit}

Based on the analysis above, the circuit is designed as shown in Figure VII. In this circuit, in addition to including the main rectifier filter and BUCK form DC-DC circuit, but also includes two parts of the control circuit: overvoltage protection circuit and DC-DC start control circuit.

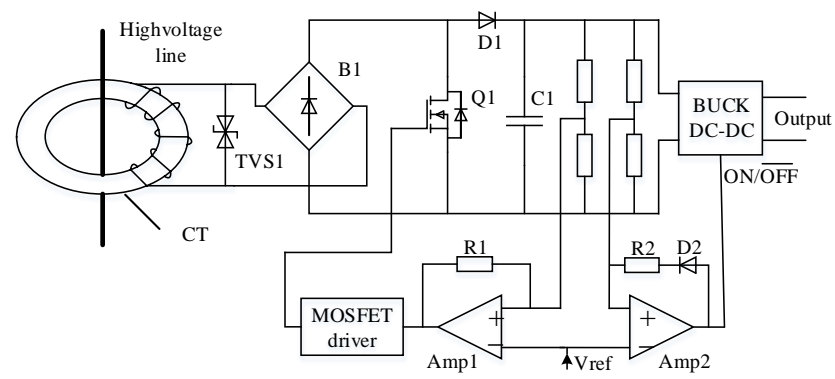

FIGURE VII. SCHEMATIC DIAGRAM OF HARDWARE CIRCUIT

The role of DC-DC start control circuit is to make the power supply directly across the area I and works in area II (reasons see below). Through the control of hysteresis comparator composed by amplifier Amp2, at the rising stage of the voltage of the capacitor $\mathrm{C} 1$, control the DC-DC circuit in off state. At this time, the DC-DC circuit does not output voltage, and its own standby current is very small, the output current of CT will quickly charge $\mathrm{C} 1$ to a high voltage(higher than the voltage corresponding to maximum power point). At this time, start the DC-DC circuit and then the circuit will automatically reach to the power balance, and the working point can reach to the maximum power point. When the power is no longer balanced and the voltage of capacitor $\mathrm{C} 1$ drops to the voltage under the minimum input voltage of DC-DC circuit, switch the DC-DC circuit off. This can avoid outputting voltage to the load below its rated value, so as not to cause abnormal operation of the device.

In the circuit, operational amplifier Amp1 is used for overvoltage protection. When the line current is large or the power consumption is relatively small, in order to prevent the voltage of $\mathrm{C} 1$ from exceeding limit and damage the circuit, and also prevent the magnetic saturation of the iron core, turn on Q1 to discharge the output current of CT. The power supply of amplifier and other devices is provided by capacitor $\mathrm{C} 1$ through voltage regulator LM317. The DC-DC circuit is composed of LM2596, and its operating voltage is $4.5-40 \mathrm{~V}$. It can be turn on or off by controlling the voltage of pin 5 .

\section{EXPERIMENTAL RESULTS}

Use the CT designed above, the DC-DC circuit fixed output $5 \mathrm{~V}$. The minimum required line current is measured when the circuit can output $0.5 \mathrm{~W}$ and $1 \mathrm{~W}$ power under different turns. The results are shown in Table I. It can be seen from the table that the minimum required current is very close when the turn number is less than 120. Considering the experimental error and the conversion efficiency of DC-DC circuit, it can be seen that the maximum output power of $\mathrm{CT}$ is independent of the turn number. When the turn number is more than 160 , due to the voltage limit of the DC-DC circuit, the CT cannot work at the maximum power output point, the required line current is larger. Thus, the selection of 120 turns is reasonable.

\section{TABLE I. EFFECT OF TURNS ON THE OUTPUT POWER}

\begin{tabular}{|c|c|c|c|c|c|c|c|}
\hline \multicolumn{2}{|c|}{ Turns } & 60 & 80 & 100 & 120 & 160 & 200 \\
\hline \multirow{2}{*}{$\begin{array}{c}\text { Minimum } \\
\text { required } \\
\text { current (A) }\end{array}$} & $0.5 \mathrm{~W}$ & 7.5 & 7.5 & 7.6 & 7.7 & 8.5 & 9.4 \\
\cline { 2 - 8 } & $1 \mathrm{~W}$ & 11.3 & 11.3 & 11.5 & 11.6 & 13.4 & 14.5 \\
\hline
\end{tabular}

In order to verify the effect of the DC-DC start control circuit, first test the result when this part circuit is not added. The DC-DC circuit works when there is an input voltage. The output voltage of DC-DC circuit is $5 \mathrm{~V}$ and the load is $50 \Omega$. It is equivalent to a constant power $(0.5 \mathrm{~W})$ load when it is working.

The measured waveform is shown in Figure VIII (a) when the primary side current is 10A RMS. It can be seen that the filter capacitor voltage is lower than the minimum input voltage of DC-DC circuit, the DC-DC circuit cannot output the normal voltage, and has no voltage regulating ability. When the voltage of the filter capacitor increases small, the power consumption of the load increases too, then the voltage is pulled down, and finally the voltage is stabilized at a low value. As the filter capacitor voltage is low, the output power of CT is small, the circuit is not able to cross the maximum power point and achieves a new balance of power. When the primary side current increases to $16 \mathrm{~A} \mathrm{RMS}$, the voltage on the filter capacitor can rise to high enough to meet the power requirements of the load, as shown in Figure VIII (b). 


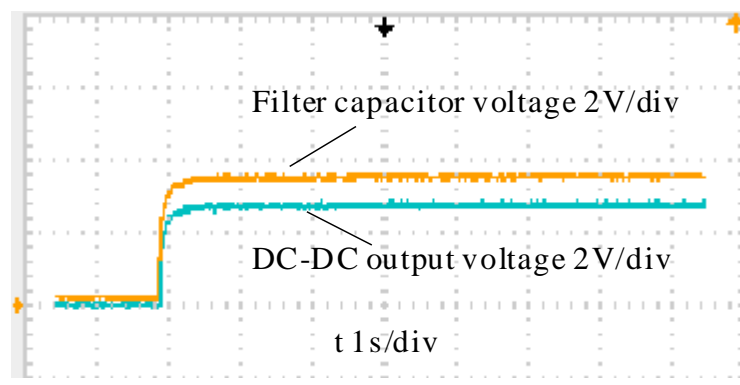

(a) $\mathrm{I} 1=10 \mathrm{~A}$ RMS

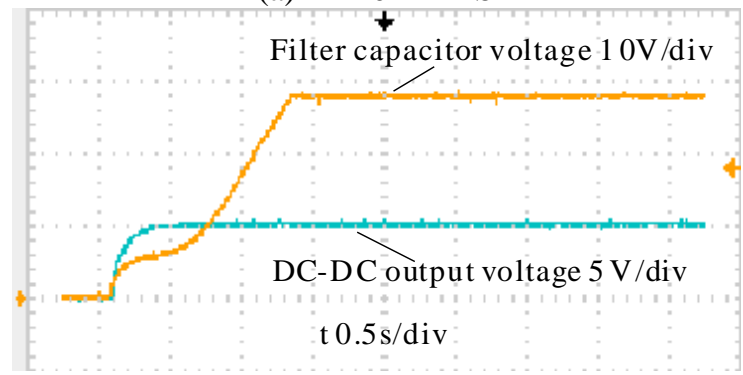

(b) I1 $=16 \mathrm{~A}$ RMS

FIGURE VIII. FILTER CAPACITOR AND DC-DC OUTPUT VOLTAGE (DC-DC NO CONTROL)

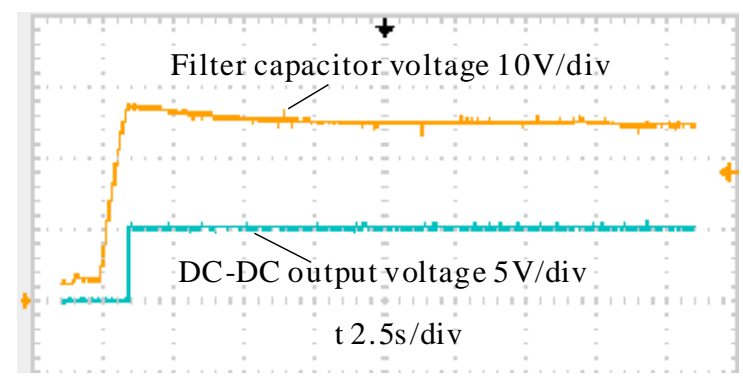

FIGURE IX. FILTER CAPACITOR AND DC-DC OUTPUT VOLTAGE (DC-DC UNDER CONTROL)

When the DC-DC start control circuit is added, the measured waveform is shown in Figure IX when the line current is 9A RMS. It can be seen that, the filter capacitor voltage has crossed the value corresponding to the maximum power point when the DC-DC circuit starts. The filter capacitor voltage drops slightly and finally stabilized. The circuit is adjusted in the direction of increasing the output power, and finally achieves the power balance.

By comparison it is found that adding DC-DC starting control circuit is necessary. Because there is the circumstance that the line current is so small that the CT power supply cannot work. When the line current starts to rise, if the DC-DC circuit is in working state, the condition is the same as in Figure VIII. This is equivalent to increase the current dead zone. The problem can be solved by adding this part of circuit.

\section{CONCLUSION}

(1) The output power of CT first increases and then decreases with the increase of the filter capacitor voltage. There is a maximum power output point, and the maximum power is independent of the turn number when the voltage drop of the rectifier is ignored. When considering the voltage drop, the maximum power increases with the increase of turn number, but becomes saturated in the end.

(2) The CT power supply has the characteristic of automatic power balance when the filter capacitor voltage is higher than the voltage corresponding to maximum power point. By adding the DC-DC start control circuit can make the CT power supply works well in the area of current dead zone.

\section{REFERENCES}

[1] Bojie Sheng, Wenjun Zhou, "Ultra-low Power Wireless-Online-Monitoring Platform for Transmission Line in Smart Grid, " High Voltage Engineering and Application (ICHVE), pp. 244-247, October 2010.

[2] Zhimin He, Shiguang Nie, Guangyu Qu, Yadong Liu, Gehao Sheng, and Xiuchen Jiang, "The design of CT energy harvesting power supply based on phase-controlled method" Power and Energy Engineering Conference (APPEEC), pp. 1-5, March 2012.

[3] Shouqiao Xin, Liye Xiao, Guomin Zhang, Jiaxing Zhai. "Power Supply Based on Small Current Transducer for Wireless Sensor in Smart Grid, "Electrical and Control Engineering (ICECE), pp. 390-3993, June 2010.

[4] Jie Chen, Rongge Yan, Wenrong Yang, Hongyu Zhao, "A Power-line Energizing System for Active Power Electronic Current Transformer," Electrical Machines and Systems (ICEMS), pp. 4431-4435, October 2008.

[5] Zhigang Ding, Yulin Hu, Hualiang Zhou, et al. A Novel Converter Draw-out Power Circuit [J]. Automation of Electric Power Systems, 2013 , 37(12):109-113.

[6] Lan Xiong, MinJie Xu, YouZhong He, DaoJun Song, YanLong Zhao, Wei He. "A novel energy obtaining system for on-line monitoring devices of transmission line," Electrical and Control Engineering (ICECE), pp. 4300-4304, September 2011

[7] Liming Wang, Haidong Li, Changlong Chen, et al. A Novel Online Energy Extracting Device with Low Lower Limit Deadband on Transmission Line [J]. High Voltage Engineering, 2014, 40(2):344-352.

[8] Tianchen Yue, Yadong Liu, Zhimin He, et al. Power Output Characteristics of Magnetic Core in CT Energy Harvesting Devices [J]. High Voltage Apparatus, 2015, 51(1):18-23.

[9] Jiles D C, Atherton D L. Theory of ferromagnetic hysteresis [J]. Journal of Magnetism and Magnetic Materials, 1986, 61(1-2): 48-60. 